\title{
Anesthetic Management of a Patient with Coffin-Siris Syndrome
}

\author{
Deepa Divakar ${ }^{1}$, Pravin Ubale ${ }^{2}$, Pinakin Gujjar ${ }^{3}$
}

\begin{abstract}
Coffin-Siris syndrome (CSS) is a rare genetic condition with characteristic musculoskeletal abnormalities and growth restrictions, coarse facial features, feeding abnormalities, systemic malformation, and developmental and cognitive delay. ${ }^{1-3}$ Main anesthetic concerns should be difficult airway that tends to get worse with age, risk of aspiration, and periop pulmonary complications in uncooperative and mentally retarded patients. We report a case of 9-years-old male patient with CSS for dental extraction and restoration under general anesthesia.

Keywords: Coarse facial features, Coffin-Siris syndrome, Difficult airway.

Research and Innovation in Anesthesia (2019): 10.5005/jp-journals-10049-0061
\end{abstract}

\section{INTRODUCTION}

Coffin-Siris syndrome (CSS) is a rare genetic condition with an incidence of around 70 reported cases ${ }^{4}$ but a potential association of the condition with difficult airway makes such cases challenging for the anesthesiologist. This condition is characterized by short fifth (pinky) fingers, absent or hypoplastic toenails and coarse facial features like microcephaly, thick and bushy eyebrows and eyelashes, sparse facial hair, wide mouth and prominent lips, macroglossia, wide nose with flat nasal bridge. We aim to report our anesthetic experience of a 9-year-old male patient with CSS posted for dental extraction and restoration for multiple dental caries.

\section{Case Description}

A 9-year-old male patient, weighing $15 \mathrm{~kg}$, a k/c/o of CSS was posted for multiple dental extractions and restoration. Patient was born out of a nonconsanguineous marriage. It was a full-term normal vaginal delivery, and the patient cried immediately after birth. Medical history revealed a history of generalized tonic-clonic convulsions for 8 months. Patient was put on syp. phenytoin $5 \mathrm{~mL}(90 \mathrm{mg} / 5$ $\mathrm{mL}$ ) and has been seizure-free for 2 months. There was no previous history of surgery or anesthesia. At present mother complained of no nasal discharge but gave a history of similar complaints on and off since childhood. She also gave a history of feeding difficulty because of the generalized developmental delay and presently also due to the gum hypertrophy.

Preoperative examination revealed characteristic coarse facial features like microcephaly, sparse scalp hair, thick and bushy eyebrows, long eyelashes, wide nose, coarse and abundant facial hair, wide mouth, prominent and thick lips (Fig. 1). Patient also had gross painless gingival hyperplasia, which per se is not a characteristic of the condition but could have been due to valproate side effects, which the patient was on for the past 6 months (Fig. 2).

Musculoskeletal examination revealed short fifth fingers (Figs 3 and 4), and generalized hypotonia was found. Per abdomen, examination revealed umbilical hernia. Patient had a generalized developmental and cognitive delay and had intermittent aggressive behavior. A proper airway examination was not possible but wide and thick lips, a large tongue, and the gingival hypertrophy was duly noted, and a difficult airway (ventilation and intubation) was anticipated.

\footnotetext{
${ }^{1-3}$ Department of Anaesthesiology, Topiwala National Medical College and BYL Nair Hospital, Mumbai, Maharashtra, India
}

Corresponding Author: Pravin Ubale, Department of Anaesthesiology, Topiwala National Medical College and BYL Nair Hospital, Mumbai, Maharashtra, India, Phone: +91 9322211472, e-mail: drpravinubale@ gmail.com

How to cite this article: Divakar D, Ubale P, Gujjar P. Anesthetic Management of a Patient with Coffin-Siris Syndrome. Res Inno in Anesth 2019;4(1):16-18.

Source of support: Nil

Conflict of interest: None

Routine biochemical examination was within normal limits. Chest X-ray and echocardiography showed no abnormalities. EEG was within the normal limit for the age. Ultrasonography of the abdomen showed umbilical hernia. A high risk written wellinformed consent was taken, and a morning dose of antiepileptic instructed to be taken early in the morning of the surgery. Starvation confirmed on the day of surgery. Difficult airway accessories in the form of various sizes of facemasks, oral and nasopharyngeal airways, various sizes of endotracheal tubes, Proseal laryngeal mask airway (LMA), and ENT standby including tracheostomy was kept ready.

The patient was mildly sedated outside the theatre for separation from the parent with inj. ketamine $5 \mathrm{mg} / \mathrm{kg}$ i.m. intravenous cannulation was secured in the left forearm after prior application of topical prilocaine $2.5 \%$ and lignocaine $2.5 \%$ ointment. Once on the table, patient was premeditated with inj. glycopyrrolate $0.04 \mathrm{mg}$, inj. midazolam $0.5 \mathrm{mg}$, and inj. fentanyl $20 \mu \mathrm{g}$. Anti-aspiration prophylaxis was given with inj. ondansetron and inj. ranitidine intravenously prior to induction. Patient was planned for nasal intubation for dental extractions. Patient was induced with thiopentone sodium $50 \mathrm{mg}$ slowly intravenously. Patient could not be adequately ventilated with facemask due to his coarse facial features and gingival hypertrophy. A larger facemask too was found, inadequate as it caused a leak from the lower part. Use of oropharyngeal airway was deferred, in view of the gross gingival hypertrophy and chance of injury and bleeding. Instead, a nasopharyngeal airway was inserted, and leak in the facemask 


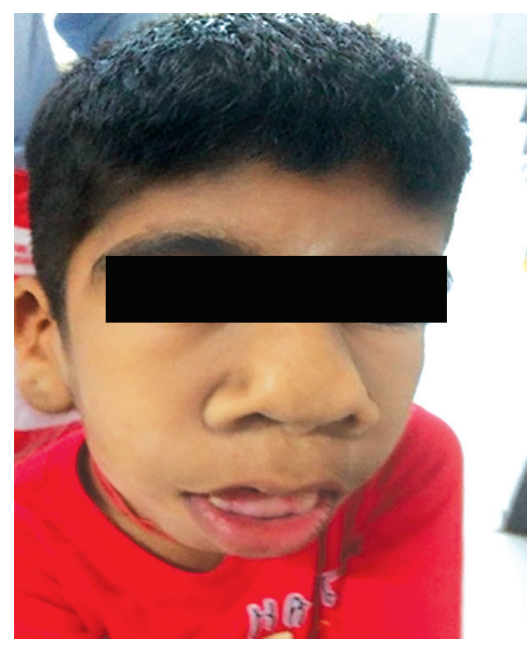

Fig. 1: Facial features of Coffin-Siris syndrome

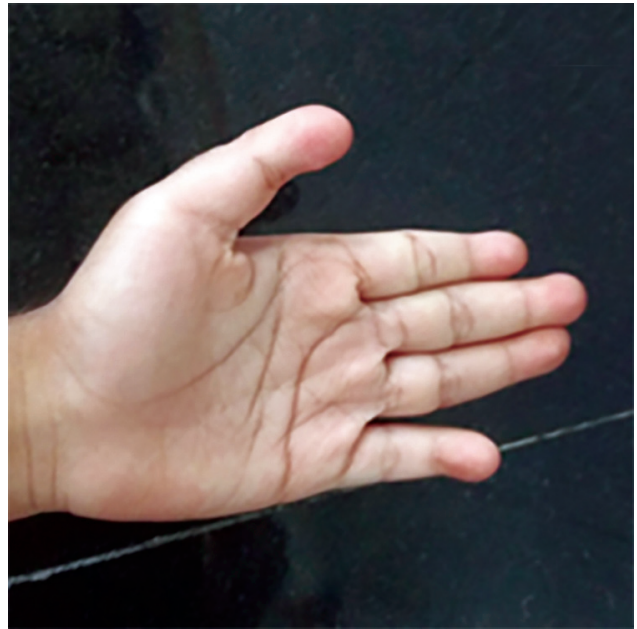

Fig. 3: Hypoplasia of phalanx of fifth finger

during ventilation was sealed with gauze pieces. Triple maneuver of chin lift, head tilt, and jaw thrust was used to facilitate adequate ventilation. Once ventilation established, inj. atracurium $7.5 \mathrm{mg}$ was given. Careful laryngoscopy was done to cause no or minimal injury to lips and gums, Cormack-Lehane grade III was observed. Cuffed endotracheal tube no. 5.0 was inserted through the nostril and negotiated through the cords with external laryngeal manipulation. Oropharyngeal throat packing was done.

Anesthesia was maintained with $\mathrm{O}_{2}$, air, and sevoflurane with intermittent doses of inj. atracurium. Hemodynamic and other vital parameters were stable. For pain relief, inj. paracetamol $150 \mathrm{mg}$, and local anesthesia were given. Delayed recovery from general anesthesia was noted. Total duration of surgery was 2 hours. Child was reversed with inj. glycopyrrolate $1.2 \mathrm{mg}$, inj. neostigmine $0.75 \mathrm{mg}$ and extubated after regular respiration and return of adequate tone, power, and reflexes. Patient was shifted to the post-anesthesia care unit (PACU) for monitoring.

\section{Discussion}

Coffin-Siris syndrome is a rare genetic and rare congenital malformation syndrome. The disorder may be characterized by intellectual disability, delay in motor skills and speech, small

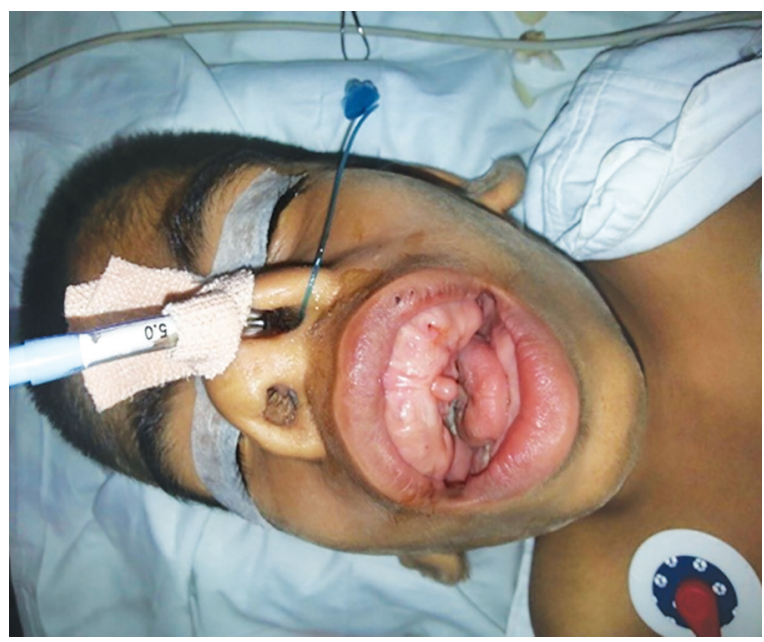

Fig. 2: Gingival hyperplasia

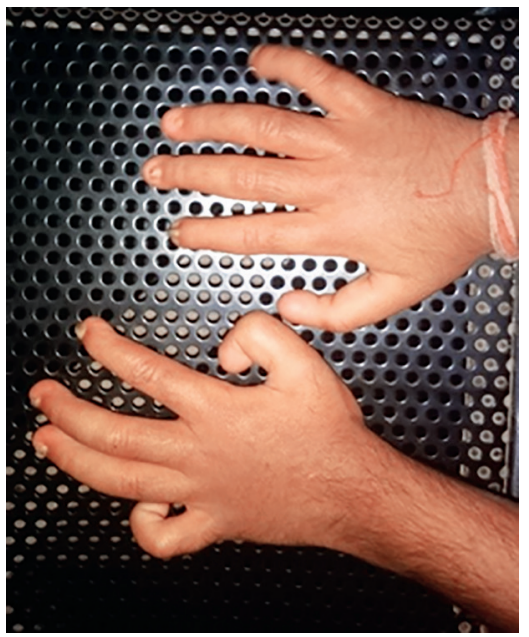

Fig. 4: Aplasia of nails on fingers

head (microcephaly), a wide nose with a low nasal bridge, a wide mouth with thick prominent lips, thick eyebrows and eyelashes (hypertrichosis), extra hair growth on the face and body, short or hypoplastic fifth fingers and eye abnormalities. Feeding difficulties and recurrent respiratory tract infections are frequent during infancy with the CSS.3 30\% of the reported case of CSS presents with congenital heart diseases. ${ }^{4}$

Seven different genes ARID1A, ARID1B, SMARCA4, SMARCB1, SMARCE1, SOX11, and PHF6 are responsible for CSS. Inheritance mode of CSS believed to be transmitted genetically as an autosomal dominant trait. ${ }^{5}$ The differential diagnoses include DOOR syndrome, hypothyroidism, mucopolysaccharidosis, and Cornelia de Lange syndrome. Patients with CSS pose many anesthetic difficulties due to the multisystem, involvement, difficult airway, developmental disabilities, and recurrent respiratory infections. Craniofacial features are unremarkable during infancy but became dysmorphic as the child grew. It is suggested that the structural changes in facial anatomy in these patients as the age increases lead to an increased likelihood of a difficult airway, and hence even a patient with a history of previous uneventful anesthesia could present with difficult airway in later surgeries.

As our patient was uncooperative, aggressive, and labile, we faced difficulties in the preoperative examination, pulmonary 
function testing, blood sampling, X-rays, and intravenous cannulation. Difficult airway in CSS may be due to microcephaly, macroglossia, gingival hyperplasia, and lax joints. Gingival hyperplasia in our patients could be due to phenytoin ${ }^{6}$ and poor oral hygiene. Manifestations of gingival enlargement usually appear within 1 months to 3 months after the start of the treatment. ${ }^{7}$ Three major drugs causing gingival hyperplasia are anticonvulsants, calcium channel blockers, and immunosuppressants.

As our patient had a history of recurrent upper respiratory infections, care was taken to prevent any form of respiratory infection by adequate nebulization and antibiotic cover. Awake fibreoptic intubation could not be done in this patient due to severe mental retardation. We observed delayed recovery from anesthesia in spite of the use of sevoflurane as an inhalational agent and shortacting atracurium as a neuromuscular relaxant.

\section{ConcLusion}

Anesthetic management of CSS is challenging due to the multisystemic nature of the disease and potential airway difficulty that could be encountered. Successful management of such cases requires meticulous preanesthetic evaluation of risk factors, the anticipation of difficult airway, and appropriate preparation and management for the same with vigilant postoperative monitoring.

\section{References}

1. Fleck BJ, Pandya A, Vanner L, et al. Coffin-Siris syndrome: review and presentation of new cases from a questionnaire study. Am J Med Genet 2001;99(1):1-7. DOI: 10.1002/1096-8628(20010215)99:1<1::aidajmg1127>3.0.co;2-a.

2. Schrier SA, Bodurtha JN, Burton B, et al. The Coffin-Siris syndrome: a proposed diagnostic approach and assessment of 15 overlapping cases. Am J Med Genet A 2012;158A(8):1865-1876. DOI: 10.1002/ ajmg.a.35415.

3. Bodurtha J, Kessel A, Berman W, et al. Distinctive gastrointestinal anomaly associated with Coffin-Siris syndrome. J Pediatr 1986;109(6):1015-1017. DOI: 10.1016/s0022-3476(86)80288-8.

4. Nemani L, Barik R, Patnaik AN, et al. Coffin-Siris syndrome with the rarest constellation of congenital cardiac defects: a case report with review of literature. Ann Pediatr Cardiol 2014;7(3):221-226. DOI: 10.4103/0974-2069.140859.

5. Carey JC, Hall BD. The Coffin-Siris syndrome: five cases including two siblings. Am J Dis Child 1978;132:667-671. DOI: 10.1001/ archpedi.1978.02120320027005.

6. Dongari-Baqtzoglou A, Research, Science and Therapy Committee, American Academy of Periodontology. Drug-associated gingival enlargement. J Periodontol 2004;75(10):1424-1431. DOI: 10.1902/ jop.2004.75.10.1424.

7. Newman MG, Takei H, Klokkevold PR, et al. Carranza's Clinical Periodontology, 10th ed., St. Louis: Saunders, Elsevier; 2006. pp. 375-376. 